\title{
Plus de 1350 soutiens-gorges à la place de la Confédération le 20 octobre 2008
}

\section{Comité de la Société Suisse de Sénologie (SSS)*}

\section{* Comité de la SSS: Christoph Rageth, Zurich (président); Bernhard Allgayer Lucerne; Gilles Berclaz, Berne; Rosmarie Caduff, Zurich; Elena Cauzza, Bellinzone; Jean-François Delaloye, Lausanne ; Véronique Dupont-Lampert, St-Gall; Silvia Ess, St-Gall; Günther Gruber, Zurich ; Felix Haberthür Binningen; Anton Haid, A-Feld- kirch; Walter R. Marti, Bâle; Hans-Jakob Müller, Bâle; Christian Öhlschlegel, St-Gall; Brigitte Pittet, Genève; Christoph Rochlitz, Bâle; Rolf Steiner, Coire; Beat Thürlimann, St-Gall; Regula Umbricht, Zurich; Carsten Viehl, Bâle; Georges Vlastos, Genève; Dieter Wallwiener, D-Tübingen; Markus Zuber, Olten.}

Traduction: Margit Eisele Bachelard, Genève
Une fois de plus, une manifestation est organisée sur la place de la Confédération. Un soutien-gorge sera suspendu par une ficelle le 20 octobre prochain, représentant chaque femme décédée d'un cancer du sein au courrant de l'année passée. Cette manifestation est à l'initiative de femmes militant pour un programme de dépistage systématique du cancer du sein par mammographie. C'est l'occasion de nous demander pourquoi ce genre de manifestations doit avoir lieu en Suisse, alors que dans tous les pays voisins ce genre de programmes existe déjà.

Il y a environs 10 ans débutait le screening mammographique sur un mode biannuel pour les femmes de 50 à 70 ans; il semblait sur la bonne voie. Or en 2001 une étude (Gotzsche et Olsson [1]) a critiqué ce dépistage au travers d'une publication très questionnable, aboutissant à interruption des tentatives d'introduction de ce dernier. La motivation des auteurs est incertaine, sachant qu'avant et après leur étude l'efficacité du screening mammographique a été corrélée avec une diminution de mortalité se situant aux alentours de $25 \%$ en population générale, atteignant 30-35\% dans le groupe des femmes participant régulièrement au dépistage. La réduction de cette mortalité pourrait donc être encore plus importante en incluant dans le programme les personnes peu sensibles aux questions de santé, qui péjorent actuellement la mortalité par cancer du sein. D'ailleurs, les taux de mortalité par cancer mammaire des Pays-Bas - qui a introduit le dépistage dans les années 90 - montre aujourd'hui une diminution significative (fFig. 1).

Malgré le fait que la diminution de la mortalité du cancer du sein grâce au screening mammographique ne soit plus mise en question, et que le conseiller fédéral Pascal Couchepin ait fait un appel aux cantons en faveur de l'introduction du dépistage, il reste plusieurs points critiques montrant la nécessité d'un contrôle de qualité et d'une bonne information des femmes invitées au screening.

\section{Etudes faibles}

Certaines études isolées n'ont pas les mêmes résultats positifs que la plupart des autres. En particulier au Canada, la supériorité de la mammographie n'a pas pu être prouvée. Cette étude s'ap- puyait cependant sur technologie radiologique aux dessous des exigences techniques adéquates. Malgré ça, elle est préférentiellement citée par les opposants au dépistage, ces derniers négligeant le fait que quasiment toutes les autres études existantes montrent des avantages manifestes, avec des baisses de mortalité allant jusqu'à 40\% [2] .

\section{Fausse alerte}

\section{Résultats mammographiques = faux positifs} et par conséquent biopsies inutiles?

Effectivement, la mammographie n'est pas une méthode parfaite. En cas de situation peu claire, la démarche diagnostique suivante passe généralement par une échographie. Si la situation reste encore incertaine, la plupart des cas sont suivis par une biopsie. Rares sont les cas ou la nécessité d'une biopsie ouverte en anesthésie générale s'impose aujourd'hui, car la plupart des diagnostics peuvent être obtenus avec une biopsie à l'aiguille fine en ambulatoire, sous anesthésie locale. Avec l'expérience d'aujourd'hui on peut dire qu'environ $5 \%$ des examens de dépistage induisent des examens supplémentaires. Ces exa-

\section{Figure 1}

Comme attendu, la mortalité du cancer du sein a été abaissée d'environ 25\% au Pays-Bas (ligne bleue) correspondant ainsi aux valeurs attendues avec dépistage (ligne rouge) - et à la différence des chiffres sans dépistage (ligne verte) (avec permission de Jacques Fracheboud, Université Erasmus MC, Rotterdam).

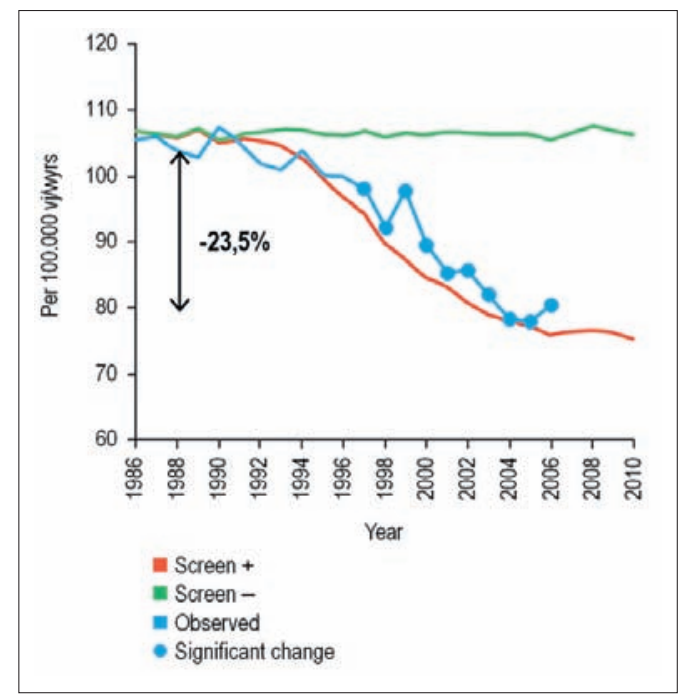


mens supplémentaires provoquent certes des angoisses supplémentaires, mais à contrario, les résultats rassurants d'un dépistage normal calment 95\% des femmes investiguées. C'est notamment pour éviter un chiffre trop élevé de fausses alertes que l'âge du début du dépistage a été fixé à 50 ans, et qu'un contrôle de qualité encadre obligatoirement le programme de dépistage.

\section{Surdiagnostic}

C'est le cas quand une femme n'aurait jamais dans sa vie compris qu'elle avait un cancer du sein en absence du dépistage.

La découverte d'un cancer du sein par mammographie ne permet pas encore des conclusions sur sa probable croissance. Ainsi il n'aurait peutêtre jamais atteint la taille permettant sa détection par la palpation dans les 10-20 ans. Les chiffres suggèrent que le taux de surdiagnostic se situe entre 5 et $10 \%$. La mortalité naturelle sans dépistage du cancer du sein est de $40 \%$ (au minimum 40 décès sur 100 femmes atteintes du cancer du sein) et la réduction de la mortalité grâce au programme de dépistage mammographique se situe aux alentours de $25 \%$ (environs 10 sur 40 ). Donc, pour une vie de femme sauvée il y a environs une femme qui souffre d'un diagnostic «inutile».

C'est le prix à payer tant que nous ne savons pas interpréter les images mammographiques dans le sens de la virulence d'une tumeur. Nous ne pouvons pas non plus prévoir quelle femme va décéder d'une autre maladie ou d'un accident dans l'année à venir. En conséquence, pour éviter un surdiagnostic trop élevé, la limite supérieure de l'âge de dépistage est fixée à 70 ans.

\section{La mammographie n'évite pas le cancer du sein mais elle le découvre plus tôt} Cette postulation n'est pas juste à 100\% . Avec la découverte précoce d'un carcinome-in-situ ou un autre précurseur du carcinome invasif, ce dernier peut être évité par un traitement ciblé. Les carcinomes-in-situ représentent environs 20\% des tumeurs détectées par le screening. La situation est donc comparable à celle du dépistage des néoplasies du col utérin, où le dépistage des précurseurs et leur traitement contribue très fortement à diminuer les cancers invasifs.

\section{Espérances exagérées des femmes, comme motivation de participer au dépistage}

Pour prouver son efficacité, une participation de beaucoup de femmes a effectivement été utile. Cette efficacité n'est aujourd'hui plus mise en doute. Il reste à éliminer une situation injuste, celle de beaucoup de femmes qui souhaitent avoir l'accès à un screening de qualité, particuliè- rement après avoir bénéficié des explications en sa faveur par les professionnels de la santé, et qui constatent malheureusement qu'un tel programme n'est pas à leur disposition.

\section{Cancers ratés}

La mammographie n'est pas encore une méthode parfaite, mais durant les dernières années il y a eu beaucoup de progrès technique et on en attend encore d'autres. Le taux de détection se situe actuellement vers $80 \%$ et il est probable qu'il augmente encore avec les améliorations techniques. Les bons résultats du dépistage mammographique (taux de mortalité abaissés de $25 \%$ ) datent des années 80 , où la technique était encore nettement inférieure à celle d'aujourd'hui.

\section{Exposition aux rayons $X$}

Pour chaque examen radiologique une certaine dose de rayons X est nécessaire. La dose actuellement utilisée avec les appareils modernes et digitaux peut être négligé en comparaison avec les effets positifs du dépistage. De plus, le tissu mammaire n'est presque plus sensible aux effets néfastes des rayons X à partir de l'âge de 20 à 30 ans.

\section{Utilité surestimée}

«Seulement» $6 \%$ des femmes entre 50 et 69 ans tombent malades d'un cancer du sein et «seulement" $0,8 \%$ des femmes de la même tranche d'âge meurent pour cette raison. Pour 100 femmes subissant un dépistage mammographique par année, "seulement» 2 en profitent et 6 meurent quand même du cancer du sein. Pour sauver 1 femme d'un cancer du sein il faudra 2500 mammographies chez des femmes en bonne santé.

Ces chiffres sont corrects. Le cancer du sein reste néanmoins la tumeur maligne la plus fréquente chez la femme et est responsable de la plupart des années de vie perdues chez le groupe des femmes de moins de 70 ans. En parlant de «seulement» $0,8 \%$ de décès on banalise le chiffre des 1300 cas de décès en Suisse par année. La mammographie a le potentiel d'empêcher chaque quatrième décès lié au cancer du sein. Ce potentiel doit être utilisé. Il ne s'agit pas tellement d'empêcher les décès mais d'empêcher les décès précoces.

D'autre part il est crucial pour les femmes et leurs proches que le traitement d'une maladie précocement découverte soit moins agressif que le traitement utilisé pour des tumeurs de taille moyenne. On peut ainsi attendre du dépistage une fréquence plus élevée de thérapies conservatrices des seins, d'opérations du ganglion sentinelle au lieu de curage axillaire classique, ainsi qu'une réduction du recours à la chimiothérapie. 


\section{Profit pour les instituts qui effectuent les mammographies?}

C'est le cas contraire. Les tarifs pour les mammographies en dehors des programmes de screening sont plus élevés. Les prestations en dehors des programmes n'incluent pas forcément une deuxième interprétation des images. Les exigences pour une mammographie de dépistage sont strictes en termes de qualité, comme par exemple le deuxième avis obligatoire et une formation supplémentaire pour les radiologues. $\mathrm{Si}$ les instituts radiologiques seraient uniquement intéressés par le profit financier, ils n'investissaient pas pour autant dans le dépistage.

\section{Coûts élevés}

Les coûts par année de vie gagnée se situent entre 10000 et 20000 francs. Ces chiffres sont comparables avec ceux du cancer cervical, mais moindre que pour une chimiothérapie d'un cancer mammaire. Ces coûts par année gagnée de vie sont également inférieurs à ceux des mesures prophylactiques approuvées comme l'hémodialyse ou l'emploi de statines.

A ceci s'ajoute le soi-disant screening sauvage ou incontrôlé qui coûterait selon deux études suisses [3, 4] plus cher que le dépistage mammographique réglementé.

Malgré cela, on trouve des politiciennes et politiciens cantonaux rétissants quant aux implications financières du dépistage, sachant qu'au minimum une partie des frais devraient être payés par les cantons. Pour le moment c'est en partie les femmes elles-mêmes et les caisses maladies qui financent les mammographies en dehors des programmes de dépistage.

\section{Conclusions}

En Suisse romande où, dans la plupart des cantons, des programmes pour le screening mammographique existent déjà depuis un certain temps, la mortalité du cancer du sein a régressé progressivement d'environ 3,5\% par année entre 1995 et 2005 (CI 95\%: 2,5-4,3\%). A contrario, on a observé une régression de 1,4\% seulement en Suisse alémanique (CI $95 \%$ : 0,3-2,4\%) [5].

Il est donc temps d'équilibrer cette situation injuste concernant l'accès au dépistage mammographique. Trop de femmes suisses alémaniques ont une prise en charge dépendant de leur gynécologue, du lieu de leur domicile ou encore des ressources se trouvant dans leur portefeuille. Les coûts n'augmentent pas à cause du dépistage systématique mais la qualité du suivi médical s'améliore clairement.

L'appel s'adresse donc aux cantons, pour qu'ils suivent la demande du 21 novembre 2007 du conseiller fédéral Pascal Couchepin, les intimant d'établir un dépistage mammographique organisé, avec un contrôle de qualité.

\section{Références}

1 Olsen O, Gotzsche PC. Cochrane review on screening for breast cancer with mammography. Lancet. 2001;358(9290):1340-2.

2 Duffy SW, Tabár L, Chen HH, Holmqvist M, Yen MF, Abdsalah S, et al. The impact of organized mammography service screening on breast carcinoma mortality in seven Swedish counties. Cancer. 2002;95(3):458-69.

3 Schopper D, de Wolf C. Leistungsantrag «Sicherung der Vergütung des Mammographie-Screenings im Rahmen KVG. Submitted by the Swiss Cancer League and Oncosuisse to the Federal Office of Health June 2007.

4 Moueddeb B. Coût moyen du dépistage du cancer du sein à Genève: comparaison entre deux modes de screening. Lausanne: Institut d'économie et de management de la santé; septembre 2004

5 S. Ess, St. Gallen. VSKR. Communication personnelle. 